\title{
CORRIGENDUM
}

\section{An association between fibromyalgia and the dopamine D4 receptor exon III repeat polymorphism and relationship to novelty seeking personality traits}

D Buskila, H Cohen, L Neumann and RP Ebstein

Molecular Psychiatry (2004) 9,1052. doi:10.1038/sj.mp.4001573

Correction to: Molecular Psychiatry (2004) 9, 730-731, doi:10.1038/mp.4001506.

has identified an error with the names of the authors attributed. The correct author names are above.

Following publication of the above paper, the author 